RICYDE. Revista Internacional de Ciencias del Deporte doi: $10.5232 /$ ricyde

Rev. int. cienc. deporte

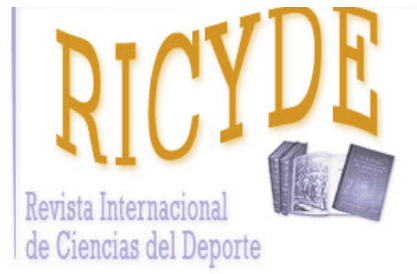

RICYDE. Revista Internacional de Ciencias del Deporte VOLUMEN XIII - AÑO XIII

Paginas:356-369 ISSN:1885-3137

Número 50 - Octubre - 2017

\title{
Niveles de ira en deportistas: diferencias en función del grado de contacto y el género \\ Anger intensity in sportspeople: differences based on contact and gender
}

\author{
José Ignacio Menéndez-Santurio y Javier Fernández-Rio \\ Universidad de Oviedo. España
}

\begin{abstract}
Resumen
Los objetivos de este estudio fueron tres: (1) analizar los niveles de ira de las personas en función del grado de contacto en el deporte practicado, (2) examinar los niveles de ira en función del género, y (3) determinar posibles correlaciones entre la ira y la edad, los años, los días y las horas semanales de práctica. 398 personas (188 hombres y 210 mujeres) entre 16 y 35 años accedieron a participar: 148 competidores de deportes de contacto, 138 de deportes sin contacto y 112 no practicantes. Se llevó a cabo una investigación cuantitativa de tipo comparativa transversal. Se utilizó el Inventario de la Expresión de la Ira EstadoRasgo. Los resultados mostraron diferencias significativas $(p<0,05)$ en el sentimiento de ira entre no practicantes y practicantes de deportes sin contacto, en la expresión verbal de la ira entre practicantes de deportes de contacto y sin contacto y en la reacción a la ira entre no-practicantes y practicantes de deportes de contacto. Asimismo, se hallaron diferencias significativas $(p<0,05)$ en las variables rasgo de ira, control interno de ira y expresión física de la ira en función del género. También se encontraron correlaciones negativas significativas $(p<0,05)$ entre la edad, días y años de práctica y la ira. Estos hallazgos señalan que los niveles de ira se ven afectados por la cantidad, intensidad y grado de contacto de su práctica deportiva, así como por el género.
\end{abstract}

Palabras clave: deportes de contacto; emoción; deportes sin contacto; psicología del deporte.

\begin{abstract}
The aims of this study were three: (1) to assess individuals' anger levels based on the contact in the sport, (2) to examine anger levels based on gender, and (3) to determine possible correlations between anger and age, years, days, and hours of practice. 398 individuals (188 males and 210 females) between 16 and 35 years agreed to participate: 148 contact-sport competitors, 138 contactless sport and 112 individuals who did not practice any sport. A comparative, transversal quantitative research design was carried out. The State-Trait Anger Expression Inventory was used. Results showed significant differences $(p<0,05)$ in anger feeling between no-practice and contactless sport athletes, in anger verbal expression between contact and contactless sport athletes and in anger reaction between no-practice and contact-sport athletes. Likewise, there were found significant differences $(p<0,05)$ in trait anger, anger control-in, and anger physical expression based on genre. There were also found significant negative correlations $(p<0,05)$ between age, days and years of practice and anger. These findings highlight that anger levels are affected by the amount, intensity and grade of contact of its practice, as well as gender.
\end{abstract}

Key words: contact sports; emotion; contactless sports; sport psychology. 


\section{Introducción}

$\mathrm{L}$ as emociones poseen un papel fundamental en el campo del deporte por su importancia para el correcto desempeño de la actividad deportiva (Poios, 2012; Wagstaff, 2014). Por ello, su estudio siempre ha tenido un rol muy significativo dentro de la investigación en el ámbito de la Psicología del Deporte (Jones, 2003).

El concepto de ira se ha utilizado intercambiablemente con las definiciones de hostilidad y agresión por las similitudes entre ambas definiciones (Carrasco y González, 2006). Debido a ello, se decidió incluir los tres términos bajo el mismo continuo al que denominaron AHA: anger (ira), hostility (hostilidad), aggression (agresión) (Spielberger, Jacobs, Russell y Crane, 1983; Spielberger y col., 1985). No obstante, los tres constructos presentan diferencias importantes. La ira se podría entender como un componente emocional formado por diferentes intensidades que oscilan desde el irritamiento hasta la furia o el enfado y suele estar unida a una activación fisiológica, neuroquímica, a una experiencia subjetiva de sentimientos $\mathrm{y}$ a un determinado modo de afrontamiento y expresión (Spielberger y col., 1985). Por su parte, la hostilidad podría definirse como una actitud negativa hacia uno o más individuos que tiene como consecuencia la emisión de un juicio adverso contra la persona (Berkowitz, 1996) y que suele producir conductas agresivas de destrucción y daño físico bien a objetos y/o personas (Spielberger y col., 1983, 1985). Por último, la agresión es aquel comportamiento cuya motivación es la de herir o hacer daño a otro individuo bien física o psicológicamente (Berkowitz, 1996). Se podría decir que la ira posee el papel emocional, la hostilidad el rol cognitivo-evaluativo y la agresión la representación conductual del continuo AHA.

Según Spielberger y col. (1985), la ira se puede analizar a partir de dos tipos principales de representación: el estado de ira y el rasgo de ira. En primer lugar, el estado de ira se conceptualizaría como la experiencia pasajera de diversos sentimientos de índole subjetiva, como la irritación o el enfado, y de forma general unida a activación fisiológica. En segundo lugar, el rasgo de ira hace mención a la predisposición de sufrir episodios de ira de forma frecuente y de diversa intensidad. Según Spielberger y col. (1998), estos acontecimientos pueden estar promovidos por diferentes causas, como la percepción de un vasto rango de situaciones como provocadoras, la propensión a experimentar situaciones negativas intensas, o también a causa de la interacción de ambos factores.

Además del estado y el rasgo, la ira posee dos manifestaciones más: la expresión y el control. Siguiendo a Miguel-Tobal, Casado, Cano-Vindel y Spielberger (2001) ambas dimensiones se subdividen en cuatro componentes: expresión interna, expresión externa, control interno y control externo. En el primer caso, el individuo que experimenta esta emoción consigue reprimirla redirigiéndola hacia su interior. En el segundo de los casos, por el contrario, la persona expresa la ira de forma externa contra objetos y/o personas de su entorno. En el tercer caso, el individuo elimina a través de la calma y el sosiego esos sentimientos mientras que, en el cuarto de los casos, la persona intenta controlar su irritación o furia tratando de evitar expresarlos contra personas y/o objetos (Miguel-Tobal y col., 2001).

La ira no ha estado exenta de su análisis y ha habido una destacable producción científica de trabajos que la han analizado en contextos deportivos (ver revisión Oliva y Fernández, 2009). Sin embargo, aunque la ira constituye una emoción muy importante en el desempeño deportivo (Nicholls, Jones, Polman, y Borkoles, 2009; Robazza y Bortoli, 2007) su análisis siempre ha tenido un papel secundario (Sofía y Fernando, 2010). Maxwell, Visek y Moores (2009) estudiaron la ira y la agresión en una muestra de competidores masculinos de cuatro deportes: baloncesto, rugby, fútbol y squash. Los resultados demostraron que el nivel de ira y 
agresión tiende a aumentar según el grado de contacto de las modalidades deportivas (mayores niveles a mayor grado de contacto). Por su parte, Boostani y Boostani (2012) compararon deportes de contacto pleno (kickboxing), controlado (karate) y no contacto (natación). Los hallazgos mostraron que el grado de contacto influía de forma significativa en los niveles de ira. Ziaee y col. (2012) analizaron los niveles de ira en adolescentes practicantes de judo, karate, natación y un grupo de no practicantes. Los hallazgos fueron contradictorios y señalaron que los karatecas y nadadores presentaban significativamente menores niveles de ira que los judocas y los no practicantes. Sofía y Fernando (2010) estudiaron la relación entre percepción de amenaza subyacente a la experiencia de estrés y ansiedad, ira y agresividad en deportes que poseían diferentes grados de contacto físico como la defensa personal, el kickboxing y el fútbol. Los resultados obtenidos mostraron que los deportistas con grados de contacto más alto (fútbol y kickboxing) presentaban mayores niveles de agresividad e ira.

En relación al género, existen muy pocos estudios que hayan analizado esta variable dentro de esta emoción. Maxwell y Morees (2007) señalaron que los atletas de género masculino y de modalidades colectivas poseen niveles superiores de ira y agresividad. Sofia y Fernando (2010) concluyeron en su estudio antes citado que las mujeres tienen tendencia a evidenciar niveles más bajos de ira y agresividad. Por su parte, Dias, Cruz y Fonseca (2014) estudiaron emociones precompetitivas en atletas masculinos y femeninos de diversos deportes (balonmano, hockey y voleibol), pero no encontraron diferencias estadísticamente significativas entre hombres y mujeres.

Los aspectos sociodemográficos de los deportistas guardan una importancia relevante a la hora de estudiar variables que influyen en el desempeño del deportista. En ese sentido, la edad, los días y las horas de entrenamiento semanales, así como los años que el deportista lleva practicando son algunas de ellas que son de especial interés.

En cuanto a la edad, los días y las horas semanales de práctica, los años de experiencia y el nivel deportivo, Menéndez y Fernández-Río (2015) encontraron en su trabajo de practicantes de boxeo y kickboxing que los atletas internacionales (los que más días y horas entrenan semanalmente) presentaban menores niveles de ira. Maxwell y col. (2009) también reflejaron que los competidores que más entrenaban, presentaban menores niveles de ira y a mayor nivel deportivo menor nivel de ira. Una de las mayores ventajas que posee el deporte es la transferencia, esto es, la posibilidad de trasladar lo que se aprende en el ámbito deportivo a entornos habituales de nuestra vida. Danish, Nellen y Owens (1996) afirman que el deporte puede producir importantes beneficios a nivel psicosocial que van más allá de lo que acontece y se aprende en contextos deportivos. En ese sentido, los autores coinciden en la necesidad de utilizar el deporte para fomentar dicha transferencia.

A pesar de que la ira ha sido estudiada en diferentes deportes de contacto (Oliva y Fernández, 2009), apenas existen trabajos que hayan analizado esta emoción según el género, el grado de contacto del deporte practicado (con contacto y sin contacto) y la comparación con personas que no practican actividad física. Los escasos trabajos que han analizado el grado de contacto y el género no lo han hecho estudiando la ira y las diferentes dimensiones que constituyen esta variable (estado, rasgo, expresión externa e interna de ira y control externo e interno de ira).

Teniendo en cuenta todo lo anterior, se plantearon los siguientes objetivos: (1) analizar los niveles de ira de las personas en función del grado de contacto en el deporte practicado, (2) examinar los niveles de ira en función del género, y (3) determinar posibles correlaciones entre la ira y la edad, los años, los días y las horas semanales de práctica. En base a estos tres objetivos se plantearon las siguientes hipótesis: (1) habrá diferencias significativas en los 
niveles de ira en función del tipo de deporte practicado, (2) habrá diferencias significativas en función del género y, (3) la edad, las horas, días y/o años de entrenamiento correlacionarán significativamente con las diferentes dimensiones de la ira.

\section{Método}

\section{Diseño y participantes}

En el estudio participaron un total de 398 hombres y mujeres entre 16 y 35 años $(\mathrm{M}=22,4$; $\mathrm{DE}=5,19)$ de los que 148 eran competidores de deportes de contacto $(\mathrm{M}=22,33 ; \mathrm{DE}=$ $3,49), 138$ eran competidores de deportes sin contacto $(\mathrm{M}=20,86, \mathrm{DE}=5,69)$ y 112 era un grupo de personas no practicantes $(\mathrm{M}=24,63, \mathrm{DE}=5,70)$. En aras de poder establecer comparaciones metodológicamente rigurosas entre los no practicantes y el resto de grupos, se seleccionaron diversos participantes del entorno familiar, laboral y de ocio de los autores del manuscrito que representaran un colectivo amplio y heterogéneo de la población general. Para poder participar en la investigación, los deportistas debían tener, como mínimo, un año de experiencia, y además, ser competidores en activo, acudiendo regularmente a los entrenamientos y competiciones pertinentes. Todos los competidores eran regionales, pertenecientes a 21 equipos en total. En el caso de los no practicantes se estableció que solo fueran considerados bajo esta denominación aquellas personas que nunca habían tenido contacto con algún tipo de actividad deportiva de manera regular. En la Tabla 1 se presenta el número y edad de los participantes. Las medias de la tabla están referidas a su promedio de edad. Se realizó una técnica de muestreo intencional (Bisquerra, 2012) donde se garantizó que todos los deportistas estaban en activo en el momento de recogida de datos. La participación fue anónima y consentida.

Tabla 1. Número y edad de los grupos participantes

\begin{tabular}{|c|c|c|c|c|c|c|}
\hline Género & Grado de contacto & Deporte & $n$ & M & DT & $\%$ \\
\hline \multirow[b]{2}{*}{$\begin{array}{l}\text { Hombres } \\
(\mathrm{n}=188)\end{array}$} & \multirow{2}{*}{$\begin{array}{c}\text { Con contacto }(n=148) \\
\text { Hombres. } n=75 \\
\text { Mujeres } n=73\end{array}$} & $\begin{array}{c}\text { Fútbol } \\
\text { Hombres. } n=41 \\
\text { Mujeres } n=43\end{array}$ & 84 & 21,3 & 3,18 & $21,1 \%$ \\
\hline & & $\begin{array}{c}\text { Baloncesto } \\
\text { Hombres. } n=34 \\
\text { Mujeres } n=30\end{array}$ & 64 & 23,5 & 3,53 & $16,1 \%$ \\
\hline \multirow{4}{*}{$\begin{array}{l}\text { Mujeres } \\
(\mathrm{n}=210)\end{array}$} & \multirow{2}{*}{$\begin{array}{c}\text { Sin contacto }(n=138) \\
\text { Hombres. } n=57 \\
\text { Mujeres } n=81\end{array}$} & $\begin{array}{c}\text { Atletismo } \\
\text { Hombres. } n=27 \\
\text { Mujeres } n=34 \\
\end{array}$ & 61 & 17,6 & 3,01 & $15,3 \%$ \\
\hline & & $\begin{array}{c}\text { Voleibol } \\
\text { Hombres. } n=30 \\
\text { Mujeres } n=47\end{array}$ & 77 & 23,4 & 5,98 & $19,3 \%$ \\
\hline & \multirow[t]{2}{*}{$\begin{array}{c}\text { No practicantes }(n=112) \\
\text { Hombres. } n=56 \\
\text { Mujeres } n=56\end{array}$} & $\begin{array}{c}\text { No practicante } \\
\text { Hombres. } n=56 \\
\text { Mujeres } n=56 \\
\end{array}$ & 112 & 24,6 & 5,70 & $28,1 \%$ \\
\hline & & Total muestra & 398 & 22,4 & 5,19 & $100 \%$ \\
\hline
\end{tabular}

Notas: $n=$ tamaño muestra, $M=$ media aritmética, $D T=$ desviación típica, $\%=$ porcentaje; las medias están referidas a su promedio de edad. 


\section{Instrumentos}

Cuestionario sociodemográfico. Se construyó con el objetivo de caracterizar a los sujetos del estudio respecto a variables como edad, género, disciplina deportiva y años, días y horas semanales de práctica.

Inventario de la Expresión de la Ira Estado-Rasgo (STAXI-2). Se utilizó el Inventario de la Expresión de la Ira Estado-Rasgo (STAXI-2) de Spielberger (1999) validado al contexto español por Miguel-Tobal y col. (2001). Este cuestionario está formado por 49 reactivos separados en 6 escalas: estado de ira, 15 ítems (p. ej. "Estoy furioso"); rasgo de ira, 10 ítems (p. ej. "Tengo un carácter irritable") expresión externa de ira, 6 ítems (p. ej. "Discuto con los demás"); expresión interna de ira, 6 ítems (p. ej. "Ardo por dentro aunque no lo demuestro"); control externo de ira, 6 ítems (p. ej. "Controlo mi comportamiento") y control interno de ira, 6 ítems (p. ej. "Pienso en algo agradable para tranquilizarme"). A su vez, la escala estado de ira está formada por 3 subescalas: sentimiento de ira, 5 ítems (p. ej. "Estoy furioso"); expresión verbal de la ira, 5 ítems (p. ej. "Me dan ganas de gritar"); expresión física de la ira, 5 ítems (p. ej. "Tengo ganas de abofetear a alguien") mientras que la escala rasgo de ira está constituida por 2 subescalas: temperamento de ira, 5 ítems (p. ej. "Soy una persona exaltada") y reacción a la ira, 5 ítems (p. ej. "Me pone furioso que me critiquen delante de los demás"). La escala, estado de ira mide intensidad y tiene una respuesta tipo Likert de 4 puntos $(1=$ no, en absoluto; $4=$ mucho). El resto de escalas evalúan frecuencia y también tienen una respuesta Likert de 4 puntos ( $1=$ casi nunca; $4=$ casi siempre $)$. Utilizando las escalas de expresión y control de la ira se puede obtener el índice de expresión de ira que proporciona una medida general para la expresión y control de la ira. En la Tabla 2 se muestran las escalas y subescalas del STAXI-2, y sus alpha de Cronbach.

Tabla 2. Escalas, subescalas y alpha de Cronbach de la escala STAXI-2

\begin{tabular}{|c|c|}
\hline Escalas & Subescalas \\
\hline Estado de ira $\alpha=0,87$ & $\begin{array}{c}\text { Sentimiento de ira } \alpha=0,77 \\
\text { Expresión verbal de la ira } \alpha=0,78 \\
\text { Expresión física de la ira } \alpha=0,78\end{array}$ \\
\hline Rasgo de ira $\alpha=0,80$ & $\begin{array}{c}\text { Temperamento de ira } \alpha=0,84 \\
\text { Reacción a la ira } \alpha=0,76\end{array}$ \\
\hline Expresión externa de la ira $\alpha=0,69$ & \\
\hline Expresión interna de la ira $\alpha=0,62$ & \\
\hline Control externo de la ira $\alpha=0,84$ & \\
\hline Control interno de la ira $\alpha=0,81$ & Índice de expresión de la ira $\alpha=0,75$ \\
\hline
\end{tabular}

\section{Procedimiento}

Primeramente, se obtuvo el permiso del Comité de Ética de la Universidad de los autores del estudio. A continuación, se contactó con los directores de los equipos participantes de la investigación para explicarles el objetivo del mismo. Tras su consentimiento, el investigador principal administró los cuestionariosal finalizar una de las sesiones de entrenamiento. En todo momento se incidió en que contestaran con la mayor sinceridad posible, informándoles de que sus datos serían tratados de forma totalmente confidencial. La duración aproximada para cumplimentar el cuestionario fue de 15 minutos. Para el caso de los no practicantes, se contactó con personas cercanas al ámbito de los investigadores que nunca habían tenido contacto con algún tipo de actividad física de forma regular. En referencia a las preguntas del cuestionario, se informó a los participantes deportistas que los diferentes ítems no estaban referidos a la competición sino a la vida diaria. 


\section{Análisis de datos}

Se llevó a cabo una investigación cuantitativa de tipo comparativa transversal en la que se ha utilizado un diseño transcultural de dos grupos diferentes: deportistas y no deportistas (Ato, López, y Benavente, 2013). Los datos fueron tratados con el programa SPSS en su versión 22,0 (IBM, Chicago, IL). En primer lugar, se realizó la prueba Kolmogorov-Smirnov para analizar la distribución de la muestra. Se obtuvieron valores $p<0,05$ en todas las variables. Eso implica que no existe una distribución normal de la muestra y que por tanto, es necesario emplear pruebas no paramétricas. En aras de conocer las posibles diferencias entre los grupos de estudio, se realizaron los pertinentes análisis de contraste utilizando la prueba no paramétrica $\mathrm{H}$ de Kruskal-Wallis. A continuación, con el objeto de conocer entre qué grupos había diferencias estadísticamente significativas, se realizó la prueba $\mathrm{U}$ de Mann-Whitney ( $p$ $<0,05)$ por parejas. Asimismo, se utilizó la prueba U Mann-Whitney $(p<0,05)$ para conocer las posibles diferencias por género entre los grupos (deportes de contacto, deportes sin contacto y no practicantes) y entre los deportes (fútbol, baloncesto, voleibol y atletismo). Se obtuvo el tamaño del efecto $\left(\mathrm{n}^{2}\right)$ de las pruebas realizadas, siendo los valores de referencia los siguientes: $0,01=$ efecto pequeño, $0,06=$ efecto mediano y $0,14=$ efecto grande. Finalmente, para establecer correlaciones entre la edad, los años, días y horas semanales de práctica y las variables de la escala se utilizaron las correlaciones Rho de Spearman $(p<0,05)$.

\section{Resultados}

Con el objetivo de conocer las diferencias entre los grupos, se realizó la prueba no paramétrica $\mathrm{H}$ de Kruskal-Wallis $(p<0,05)$. En primer lugar, se ejecutó la prueba comparando el grado de contacto (deportes con contacto-sin contacto) y el grupo de no practicantes (Tabla 3 ).

Tabla 3. Medias, desviaciones típicas y prueba Kruskal-Wallis por grupos.

\begin{tabular}{|c|c|c|c|c|c|c|}
\hline Variables & $\begin{array}{c}\text { Deportes de } \\
\text { contacto } \\
\mathrm{M}(\mathrm{DT})\end{array}$ & $\begin{array}{c}\text { Deportes sin } \\
\text { contacto } \\
\mathrm{M}(\mathrm{DT})\end{array}$ & $\begin{array}{c}\text { No } \\
\text { practicantes } \\
\mathrm{M}(\mathrm{DT})\end{array}$ & $\begin{array}{c}\text { Chi- } \\
\text { Cuadrado }\end{array}$ & Valor $p$ & $\eta 2$ \\
\hline Estado de ira & $1,64(0,43)$ & $1,55(0,39)$ & $1,63(0,38)$ & 4,543 & 0,103 & 0,011 \\
\hline Rasgo de ira & $1,98(0,57)$ & $1,92(0,52)$ & $2,10(0,48)$ & 5,556 & 0,062 & 0,013 \\
\hline Expresión externa de ira & $1,82(0,56)$ & $1,84(0,56)$ & $1,86(0,49)$ & 1,271 & 0,530 & 0,003 \\
\hline Expresión interna de ira & $2,17(0,52)$ & $2,06(0,58)$ & $2,06(0,55)$ & 5,587 & 0,061 & 0,014 \\
\hline Control interno de ira & $2,39(0,69)$ & $2,42(0,77)$ & $2,30(0,68)$ & 1,311 & 0,519 & 0,003 \\
\hline Control externo de ira & $2,88(0,78)$ & $2,85(0,78)$ & $2,78(0,73)$ & 1,244 & 0,537 & 0,003 \\
\hline Sentimiento de ira & $1,65(0,46)$ & $1,56(0,43)$ & $1,75(0,50)$ & 10,022 & 0,007 & 0,025 \\
\hline Expresión física de la ira & $1,34(0,48)$ & $1,34(0,44)$ & $1,27(0,39)$ & 2,014 & 0,365 & 0,005 \\
\hline Expresión verbal de la ira & $1,94(0,61)$ & $1,77(0,57)$ & $1,87(0,62)$ & 6,072 & 0,048 & 0,015 \\
\hline Temperamento de ira & $1,63(0,65)$ & $1,58(0,55)$ & $1,66(0,60)$ & 1,617 & 0,446 & 0,004 \\
\hline Reacción a la ira & $2,33(0,72)$ & $2,38(0,72)$ & $2,54(0,61)$ & 8,241 & 0,016 & 0,020 \\
\hline Índice de expresión de ira & $34,70(1,63)$ & $34,63(1,62)$ & $34,82(1,46)$ & 0,906 & 0,636 & 0,002 \\
\hline
\end{tabular}

Notas: ${ }^{*} p<0,05 ; M=$ media aritmética, $D T=$ desviación típica, $p=$ valor de significación. 
Posteriormente, se empleó la prueba U de Mann-Whitney para conocer entre qué grupos había diferencias estadísticamente significativas (ver Tabla 4). Se ajustó el nivel de riesgo del contraste teniendo en consideración el número de grupos a comparar $(p<0,017)$. Se encontraron diferencias estadísticamente significativas entre varios grupos en tres dimensiones de la ira: sentimiento de ira, expresión verbal de ira y reacción a la ira, expresión verbal de ira y reacción a la ira, así como en los años, días y horas semanales de práctica.

Tabla 4. Prueba U de Mann-Whitney por grupos

\begin{tabular}{|c|c|c|c|c|}
\hline Variable & Grupos & Z & Valor $p$ & $\eta 2$ \\
\hline Sentimiento de ira & Deportes sin contacto - no practicantes & $-3,119$ & 0,002 & 0,023 \\
\hline $\begin{array}{c}\text { Expresión verbal de la } \\
\text { ira }\end{array}$ & Deportes sin contacto - deportes de contacto & $-2,497$ & 0,013 & 0,021 \\
\hline Reacción a la ira & Deportes con contacto - no practicantes & -2.788 & 0,005 & 0,029 \\
\hline
\end{tabular}

Notas: $* p<0,017, p=$ valor de significación ajustado.

Tras ello, se dividió la muestra según los grupos (deportes con contacto, sin contacto y no practicantes) y se realizaron análisis $U$ de Mann-Whitney para conocer las posibles diferencias por género en cada uno de los tres grupos (Tabla 5). Las comparaciones por género (hombre-mujer) son únicamente dentro de cada grupo de análisis y por tanto, el valor $p$ se mantiene en 0,05 . Se encontraron diferencias estadísticamente significativas en las variables rasgo de ira, control interno de la ira y expresión física de la ira.

Tabla 5. Prueba U Mann-Whitney por género en los diferentes grupos

\begin{tabular}{|c|c|c|c|c|c|c|}
\hline Variables & Grupos & $\begin{array}{c}\text { Hombres } \\
\text { M (DT) }\end{array}$ & $\begin{array}{c}\text { Mujeres } \\
\text { M (DT) }\end{array}$ & Z & Valor $p$ & $\eta 2$ \\
\hline Rasgo de ira & $\begin{array}{c}\text { Deportes con } \\
\text { contacto }\end{array}$ & $1,86(0.50)$ & $2,11(0.61)$ & $-2,419$ & 0,016 & 0,014 \\
\hline Control interno de ira & $\begin{array}{c}\text { Deportes sin } \\
\text { contacto }\end{array}$ & $2,22(0,76)$ & $2,55(0,76)$ & $-2,333$ & 0,020 & 0,013 \\
\hline Expresión fis. de la ira & No practicantes & $1,35(0,44)$ & $1,20(0,31)$ & $-2,198$ & 0,028 & 0,012 \\
\hline
\end{tabular}

Notas: ${ }^{*} p<0,05 ; M=$ media aritmética, $D T=$ desviación típica, $p=$ valor de significación.

Puesto que se encontraron diferencias significativas por género en los deportes con contacto para la variable rasgo de ira y en los deportes sin contacto para la variable control interno de ira, se quiso conocer entre qué deportes específicos podía haber diferencias en función del género. Por ello, se dividió la muestra según los deportes (fútbol, baloncesto, voleibol y atletismo) y se realizó la $U$ de Mann-Whitney para cada uno de los deportes según el género. Las comparaciones por género (hombre-mujer) son únicamente dentro de cada deporte, y por tanto, el valor $p$ se mantiene en 0,05 . En la Tabla 6 solo se muestran los resultados que fueron significativos. 
Menéndez-Santurio, J. I., y Fernández-Rio, J. (2017). Niveles de ira en deportistas: diferencias en función del grado de contacto y el género. RICYDE. Revista internacional de ciencias del deporte, 50(13), 356-369. https://doi.org/10.5232/ricyde2017.05003

Tabla 6. Prueba U Mann-Whitney por género en los diferentes deportes

\begin{tabular}{|c|c|c|c|c|c|c|}
\hline Variables & Deportes & $\begin{array}{c}\text { Hombres } \\
\text { M (DT) }\end{array}$ & $\begin{array}{c}\text { Mujeres } \\
\text { M (DT) }\end{array}$ & Z Valor $p$ & $\eta 2$ \\
\hline Rasgo de ira & Baloncesto & $1,81(0,56)$ & $2,27(0,57)$ & $-3,143$ & 0,002 & 0,024 \\
\hline $\begin{array}{c}\text { Control interno de } \\
\text { ira }\end{array}$ & Voleibol & $2,22(0,80)$ & $2,61(0.71)$ & $-1,922$ & 0,045 & 0,009 \\
\hline
\end{tabular}

Notas: ${ }^{*} p<0,05 ; M=$ media aritmética, $D T=$ desviación típica, $p=$ valor de significación.

Finalmente, se calcularon las correlaciones Rho de Spearman $(p<0,05)$ entre la edad, los años, días y horas semanales de práctica y las diferentes dimensiones de la ira (Tabla 7). Se encontraron correlaciones significativas negativas entre la expresión física de la ira y la edad, entre la reacción a la ira y los días a la semana y los años de práctica, y entre el rasgo de ira y los años de práctica.

Los resultados han mostrado diferencias significativas en tres variables: en el sentimiento de ira entre los no practicantes y los que practican deportes sin contacto, en la expresión verbal de ira entre los practicantes de deportes de contacto y sin contacto y en la reacción a la ira entre los no practicantes y los que practican deportes de contacto. En función del género se han encontrado diferencias significativas en la expresión física de la ira entre los no practicantes (mayores en los hombres), en el control interno de la ira entre los practicantes de deportes sin contacto (mayores en las mujeres) y en el rasgo de la ira entre los practicantes de deportes sin contacto (mayores en las mujeres). Asimismo, según el género, entre los diferentes deportes de la muestra se han encontrado diferencias significativas en el rasgo de la ira (mayores en las baloncestistas) y control interno de ira (mayor en las jugadoras de voleibol). Finalmente se han encontrado correlaciones negativas entre la expresión física de la ira y la edad, entre la reacción a la ira y los días y años de práctica y entre el rasgo de ira y los años de práctica. 
Menéndez-Santurio, J. I., y Fernández-Rio, J. (2017). Niveles de ira en deportistas: diferencias en función del grado de contacto y el género. RICYDE. Revista internacional de ciencias del deporte, 50(13), 356-369. https://doi.org/10.5232/ricyde2017.05003

Tabla 7. Correlaciones Rho de Spearman

\begin{tabular}{|c|c|c|c|c|c|c|c|c|c|c|c|c|c|c|c|c|c|}
\hline Variables & & 1 & 2 & 3 & 4 & 5 & 6 & 7 & 8 & 9 & 10 & 11 & 12 & 13 & 14 & 15 & 16 \\
\hline 1. Edad & $\begin{array}{c}\text { C.C. } \\
p\end{array}$ & 1 & & & & & & & & & & & & & & & \\
\hline 2. Años de práctica & $\begin{array}{c}P \\
\text { C.C. } \\
P\end{array}$ & $\begin{array}{l}0,11^{*} \\
0,023\end{array}$ & 1 & & & & & & & & & & & & & & \\
\hline 3. Días sem. de práctica & $\begin{array}{c}\text { C.C. } \\
P\end{array}$ & $\begin{array}{c}0,32 * * \\
0,000\end{array}$ & $\begin{array}{c}0,62 * * \\
0,000\end{array}$ & 1 & & & & & & & & & & & & & \\
\hline 4. Horas sem. de práctica & $\begin{array}{c}\text { C.C. } \\
P\end{array}$ & $\begin{array}{c}0,28^{* *} \\
0,000\end{array}$ & $\begin{array}{c}0,63 * * \\
0,000\end{array}$ & $\begin{array}{c}0,96^{* *} \\
0,000\end{array}$ & 1 & & & & & & & & & & & & \\
\hline 5. Estado de ira & $\begin{array}{c}\text { C.C. } \\
P\end{array}$ & $\begin{array}{c}0,06 \\
0,237\end{array}$ & $\begin{array}{l}-0,02 \\
0,508\end{array}$ & $\begin{array}{l}-0,04 \\
0,365\end{array}$ & $\begin{array}{l}-0,04 \\
0,281\end{array}$ & 1 & & & & & & & & & & & \\
\hline 6. Rasgo de ira & $\begin{array}{c}\text { C.C. } \\
P\end{array}$ & $\begin{array}{l}-0,06 \\
0,197\end{array}$ & $\begin{array}{c}-0,10^{*} \\
0,039\end{array}$ & $\begin{array}{l}-0,08 \\
0,099\end{array}$ & $\begin{array}{l}-0,07 \\
0,165\end{array}$ & $\begin{array}{c}0,51 * * \\
0,000\end{array}$ & 1 & & & & & & & & & & \\
\hline 7. Expresión externa de ira & $\begin{array}{c}\text { C.C. } \\
P\end{array}$ & $\begin{array}{l}-0,06 \\
0,218\end{array}$ & $\begin{array}{l}-0,04 \\
0,346\end{array}$ & $\begin{array}{l}-0,04 \\
0,430\end{array}$ & $\begin{array}{l}-0,06 \\
0,193\end{array}$ & $\begin{array}{c}0,58 * * \\
0,000\end{array}$ & $\begin{array}{c}0,58^{* *} \\
0,000\end{array}$ & 1 & & & & & & & & & \\
\hline 8. Expresión interna de ira & $\begin{array}{c}\text { C.C. } \\
P\end{array}$ & $\begin{array}{l}-0,04 \\
0,407\end{array}$ & $\begin{array}{c}0,01 \\
0,809\end{array}$ & $\begin{array}{c}0,03 \\
0,439\end{array}$ & $\begin{array}{c}0,02 \\
0,592\end{array}$ & $\begin{array}{c}0,33^{* *} \\
0,000\end{array}$ & $\begin{array}{c}0,27 * * \\
0,000\end{array}$ & $\begin{array}{c}0,19 * * \\
0,000\end{array}$ & 1 & & & & & & & & \\
\hline 9. Control interno de ira & $\begin{array}{c}\text { C.C. } \\
P\end{array}$ & $\begin{array}{c}0,01 \\
0,758\end{array}$ & $\begin{array}{l}-0,01 \\
0,715\end{array}$ & $\begin{array}{c}0,02 \\
0,697\end{array}$ & $\begin{array}{c}0,05 \\
0,254\end{array}$ & $\begin{array}{l}-0,09 \\
0,071\end{array}$ & $\begin{array}{l}-0,10 \\
0,043\end{array}$ & $\begin{array}{c}0,16^{* *} \\
0,001\end{array}$ & $\begin{array}{l}0,15^{*} \\
0,003\end{array}$ & 1 & & & & & & & \\
\hline 10. Control externo de ira & $\begin{array}{c}\text { C.C. } \\
P\end{array}$ & $\begin{array}{c}0,01 \\
0,767\end{array}$ & $\begin{array}{c}0,00 \\
0,929\end{array}$ & $\begin{array}{c}0,02 \\
0,626\end{array}$ & $\begin{array}{c}0,02 \\
0,606\end{array}$ & $\begin{array}{c}0,28 * * \\
0,000\end{array}$ & $\begin{array}{c}0,33^{* *} \\
0,000\end{array}$ & $\begin{array}{c}0,35^{* *} \\
0,001\end{array}$ & $\begin{array}{c}0,13^{* *} \\
0.008\end{array}$ & $\begin{array}{c}0,46 * * \\
0,000\end{array}$ & 1 & & & & & & \\
\hline 11. Sentimiento de ira & $\begin{array}{c}\text { C.C. } \\
P\end{array}$ & $\begin{array}{c}0,09 \\
0,057\end{array}$ & $\begin{array}{l}-0,08 \\
0,113\end{array}$ & $\begin{array}{l}-0,09 \\
0,066\end{array}$ & $\begin{array}{l}-0,08 \\
0,106\end{array}$ & $\begin{array}{c}0,72 * * \\
0,000\end{array}$ & $\begin{array}{c}0,43 * * \\
0,000\end{array}$ & $\begin{array}{c}0,41 * * \\
0,000\end{array}$ & $\begin{array}{c}0.27 * * \\
0.000\end{array}$ & $\begin{array}{c}0,13 * * \\
0,008\end{array}$ & $\begin{array}{c}0,26^{* *} \\
0,000\end{array}$ & 1 & & & & & \\
\hline 12. Expresión física de la ira & $\begin{array}{c}\text { C.C. } \\
P\end{array}$ & $\begin{array}{c}-0,11^{*} \\
0,019\end{array}$ & $\begin{array}{c}0,01 \\
0,699\end{array}$ & $\begin{array}{c}0,04 \\
0,383\end{array}$ & $\begin{array}{c}0,03 \\
0,464\end{array}$ & $\begin{array}{c}0,67 * * \\
0,000\end{array}$ & $\begin{array}{l}0,38^{*} \\
0,000\end{array}$ & $\begin{array}{c}0,42 * * \\
0,000\end{array}$ & $\begin{array}{c}0.27 * * \\
0.000\end{array}$ & $\begin{array}{l}-0,07 \\
0,154\end{array}$ & $\begin{array}{c}0,24^{* *} \\
0,000\end{array}$ & $\begin{array}{c}0,36^{* *} \\
0,000\end{array}$ & 1 & & & & \\
\hline 13. Expresión verbal de la ira & $\begin{array}{c}\text { C.C. } \\
P\end{array}$ & $\begin{array}{c}0,09 \\
0,064\end{array}$ & $\begin{array}{c}0,01 \\
0,731\end{array}$ & $\begin{array}{l}-0,01 \\
0,799\end{array}$ & $\begin{array}{l}-0,03 \\
0,466\end{array}$ & $\begin{array}{c}0,88 * * \\
0,000\end{array}$ & $\begin{array}{c}0,41 * * \\
0,000\end{array}$ & $\begin{array}{c}0,51 * * \\
0,000\end{array}$ & $\begin{array}{c}0.28^{* *} \\
0.000\end{array}$ & $\begin{array}{l}-0,02 \\
0,572\end{array}$ & $\begin{array}{c}0,17 * * \\
0,000\end{array}$ & $\begin{array}{c}0,47 * * \\
0,000\end{array}$ & $\begin{array}{c}0,47 * * \\
0,000\end{array}$ & 1 & & & \\
\hline 14. Temperamento de ira & $\begin{array}{c}\text { C.C. } \\
P\end{array}$ & $\begin{array}{l}-0,01 \\
0,706\end{array}$ & $\begin{array}{l}-0,02 \\
0,673\end{array}$ & $\begin{array}{l}-0,03 \\
0,459\end{array}$ & $\begin{array}{l}-0,04 \\
0,394\end{array}$ & $\begin{array}{c}0,53 * * \\
0,000\end{array}$ & $\begin{array}{c}0,77 * * \\
0,000\end{array}$ & $\begin{array}{c}0,63 * * \\
0,000\end{array}$ & $\begin{array}{c}0.15^{* *} \\
0.001\end{array}$ & $\begin{array}{c}0,21 * * \\
0,000\end{array}$ & $\begin{array}{c}0,47 * * \\
0,000\end{array}$ & $\begin{array}{c}0,48 * * \\
0,000\end{array}$ & $\begin{array}{c}0,37 * * \\
0,000\end{array}$ & $\begin{array}{c}0,41 * * \\
0,000\end{array}$ & 1 & & \\
\hline 15. Reacción a la ira & $\begin{array}{c}\text { C.C. } \\
P\end{array}$ & $\begin{array}{l}-0,09 \\
0,056\end{array}$ & $\begin{array}{c}0,15^{* *} \\
0,002\end{array}$ & $\begin{array}{c}-0,10^{*} \\
0,030\end{array}$ & $\begin{array}{l}-0,07 \\
0,116\end{array}$ & $\begin{array}{c}0,32 * * \\
0,000\end{array}$ & $\begin{array}{c}0,85^{* *} \\
0,000\end{array}$ & $\begin{array}{c}0,35^{* *} \\
0,000\end{array}$ & $\begin{array}{c}0.159^{* *} \\
0,000\end{array}$ & $\begin{array}{c}0,00 \\
0,894\end{array}$ & $\begin{array}{c}-0,12^{*} \\
0,011\end{array}$ & $\begin{array}{c}0,26 * * \\
0,000\end{array}$ & $\begin{array}{c}0,27 * * \\
0,000\end{array}$ & $\begin{array}{c}0,26^{* *} \\
0,000\end{array}$ & $\begin{array}{c}0,37 * * \\
0,000\end{array}$ & 1 & \\
\hline 16. Índice de expresión de ira & $\begin{array}{c}\text { C.C. } \\
p\end{array}$ & $\begin{array}{l}-0,06 \\
0,188\end{array}$ & $\begin{array}{l}-0,01 \\
0,823\end{array}$ & $\begin{array}{l}-0,02 \\
0,648\end{array}$ & $\begin{array}{l}-0,05 \\
0,309\end{array}$ & $\begin{array}{c}0,46^{* *} \\
0,000\end{array}$ & $\begin{array}{c}0,48^{* *} \\
0,000\end{array}$ & $\begin{array}{c}0,61 * * \\
0,000\end{array}$ & $\begin{array}{c}0.27 * * \\
0.000\end{array}$ & $\begin{array}{c}0,68 * * \\
0,000\end{array}$ & $\begin{array}{c}0,77^{* *} \\
0,000\end{array}$ & $\begin{array}{c}0,40 * * \\
0,000\end{array}$ & $\begin{array}{c}0,37^{* *} \\
0,000\end{array}$ & $\begin{array}{c}0,33 * * \\
0,000\end{array}$ & $\begin{array}{c}0,58^{* *} \\
0,000\end{array}$ & $\begin{array}{c}0,26 * * \\
0,000\end{array}$ & 1 \\
\hline
\end{tabular}

Notas: $* p<0.05, * * p<0,01 ; C . C .=$ coeficiente de correlación, $p=$ valor de significación. 


\section{Discusión}

Los objetivos del presente estudio fueron tres: (1) analizar los niveles de ira de las personas en función del grado de contacto en el deporte practicado, (2) examinar los niveles de ira en función del género, y (3) determinar posibles correlaciones entre la ira y la edad, los años, los días y las horas semanales de práctica

Respecto a la primera hipótesis, los resultados han mostrado que existen diferencias significativas entre los practicantes de los diferentes tipos de deportes y de algunos de estos con los no practicantes en diferentes manifestaciones de la ira. Así, los practicantes de deportes sin contacto (voleibol y atletismo) tenían niveles significativamente menores de sentimiento de ira que los no practicantes y de expresión verbal de ira que los practicantes de deportes de contacto (fútbol y baloncesto). Estos resultados están en línea con los encontrados por Boostani y Boostani (2012), Maxwell y col. (2009) y Sofía y Fernando (2010). Puede que el contacto directo y los roces que se producen en deportes como el fútbol y el baloncesto entre deportistas durante el entrenamiento y la competición haga que estos expresen más fácilmente verbalmente su ira. Estos hallazgos concuerdan parcialmente con los reportados por Burns (2009) en los que no se encontraron diferencias significativas en el estado de la ira entre deportistas de contacto y no contacto, sí las hubo en el rasgo de la ira, siendo el grupo de deportistas sin contacto el que obtuvo menores niveles (reacción a la ira). Una posible explicación a este fenómeno es que muchos practicantes de deportes de contacto ven la ira como un facilitador del desempeño deportivo más que una desventaja (Robazza, Bertollo, y Bortoli, 2006). Respecto a la posible explicación que refleje la existencia de significativamente menores niveles en la reacción a la ira del grupo de deportes con contacto respecto al grupo de no practicantes pueda hallarse en las altas correlaciones que se produjeron entre el control externo de la ira y la reacción a la ira, al igual que en otros estudios (Menéndez y Fernández-Río, 2015). La práctica deportiva puede ser un elemento para ayudar a fomentar el control de la ira y mejorar el desempeño deportivo. Esto quiere decir que los deportistas, especialmente con el paso del tiempo, van adquiriendo estrategias que les permiten ayudar a controlar mejor sus emociones y, con ello, a reaccionar a la ira de forma menos pronunciada (Maxwell y col., 2009).

Respecto de la segunda hipótesis, los resultados señalan que existen algunas diferencias significativas en diferentes variables de la ira en función del género de los individuos. Así, las mujeres presentan niveles significativamente mayores del rasgo de ira en los deportes con contacto y de control interno de la ira en los deportes sin contacto. Parece como si la práctica deportiva elevara los niveles de ira en las mujeres. Estos hallazgos no concuerdan con los aportados por Sofía y Fernando (2010) en los que las mujeres reportaron menores niveles de ira o con los de Dias y col. (2014) u Oliva-Mendoza, Calleja y Hernández (2011) en donde no se encontraron diferencias significativas entre hombres y mujeres. Por el contrario, los varones solo presentaron niveles superiores de expresión física de la ira entre los no practicantes. La función catártica de la práctica deportiva puede dar sentido a estos resultados, ya que la liberación de tensión que provoca el ejercicio físico puede hacer disminuir los niveles de ira.

Finalmente, respecto a la tercera hipótesis, los resultados muestran que existen correlaciones significativas negativas entre diferentes variables. Por un lado, entre la expresión física de la ira y la edad, entre la reacción a la ira y los días y años de práctica, y entre el rasgo de ira y los y los años de práctica deportiva. En todos los casos, una mayor práctica deportiva lleva a niveles de ira más bajos. Estudios anteriores (Maxwell y col., 2009; Menéndez y FernándezRío, 2015) han mostrado resultados similares en los que a mayor tiempo de práctica deportiva 
y a mayor nivel competitivo, los individuos presentaban menores niveles de ira. Por lo tanto, la práctica deportiva puede ayudar a reducir los niveles de ira de los individuos.

Como limitaciones de la investigación destacamos, en primer lugar, la muestra limitada de cada uno de los grupos de estudio, lo que hace difícil realizar generalizaciones excesivas. En segundo lugar, es reseñable añadir que no se pudo controlar el momento en el que los no practicantes cumplimentaron los cuestionarios. Una mejor opción hubiera sido que todos ellos hubieran cumplimentado el cuestionario en un momento concreto del día donde pudieran presentar niveles de ira semejantes, al igual que se hizo con los diferentes grupos de deportistas (post-entrenamiento). Las futuras líneas de investigación se enfocan hacia la investigación en otro tipo de deportes de contacto (rugby, balonmano, judo...) y sin contacto (golf, beisbol, tenis...) para comprobar si los resultados en función del grado de contacto del deporte practicado se repiten. También sería interesante realizar comparativas entre deportes individuales y deportes colectivos.

Las implicaciones teórico-prácticas de esta investigación se centran en dos aspectos fundamentales. En primer lugar, la contribución al avance en el estudio de una variable que cumple un papel fundamental en el desempeño deportivo: la ira explicada desde un prisma multidimensional. Esto puede ayudar a que los profesionales de las Ciencias del Deporte no solo conozcan cómo la ira difiere según el tipo de deporte y el sexo, sino también cómo interacciona con otras de gran relevancia en esta área como son las variables sociodemográficas presentadas en esta investigación. Con ello, los investigadores podrán llevar a cabo estudios en este ámbito con mayor exactitud y detalle. En segundo lugar, estos hallazgos pueden tener importantes alcances para los entrenadores de deportes de contacto, especialmente los analizados en este trabajo, pues se ha visto que la expresión verbal de la ira, que puede afectar de forma negativa en el desempeño deportivo (insultos a árbitros, discusiones entre compañeros, etc.), es significativamente mayor en estos deportes que en los que no tienen contacto. Por tanto, los entrenadores deben conocer técnicas y estrategias que ayuden a que los deportistas de contacto tengan un mejor control emocional para un mejor rendimiento deportivo.

\section{Conclusiones}

A modo de conclusión, este estudio es de los escasos que aborda la ira desde un enfoque multidimensional haciendo distinción entre grupos de deportistas de contacto, sin contacto, en comparación con un grupo de no practicantes $y$ teniendo en cuenta variables sociodemográficas como el género, la edad, los años, días y horas de entrenamiento.

Los resultados muestran que existen diferencias significativas entre el grupo de deportistas sin contacto y el de no practicantes en la variable sentimiento de ira, entre el de deportistas de contacto y el de sin contacto en la variable expresión verbal y de la ira, y en el de deportistas de contacto y no practicantes en la variable reacción a la ira.

En el análisis por género, se encontraron diferencias significativas entre hombres y mujeres en el grupo de deportistas de contacto en la variable rasgo de ira, en el de deportistas sin contacto en la variable control interno de ira y en el grupo de no practicantes en la variable expresión física de la ira. Finalmente, se encontró que el nivel de ira de los deportistas se ve afectado por la cantidad, intensidad y grado de contacto de su práctica. 


\section{Referencias}

Ali, B.; Ichraf, A.; Khaled, T.; Liwa, M., \& Ali, E. (2013) Effect of gender and type of sport practiced on aggression and self-esteem in Tunisian athletes. IOSR Journal of Humanities and Social Science, 8(4), 74-80. https://doi.org/10.9790/0837-0847480

Ato, M.; López, J. J., y Benavente, A. (2013). Un sistema de clasificación de los diseños de investigación en psicología. Anales de Psicología, 29(3), 1038-1059. https://doi.org/10.6018/analesps.29.3.178511

Berkowitz, L. (1996). Agresión: Causas, consecuencias y control. Bilbao, España: Editorial Desclée de Brouwer.

Bisquerra, R. (coord.) (2012). Metodología de la investigación educativa. Madrid: La Muralla.

Bolgar, M.; Janelle, C., \& Giacobbi, P. R. (2008). Trait anger, appraisal, and coping differences among adolescent tennis players. Journal of Applied Sport Psychology, 20(1), 73-87.

https://doi.org/10.1080/10413200701790566

Boostani, M. A., \& Boostani, M. H. (2012). Investigation and comparing aggression in athletes in non-contact (swimming), limited contact (karate) and contactable (kickboxing) sport fields. Journal of Combat Sports and Martial Arts, 2(2), 87-89. https://doi.org/10.5604/20815735.1047653

Burns, D. (2009). The experience and expression of anger and aggression in dating relationships for male college athletes in contact and non-contact sports. Tesis doctoral, Faculty of the Graduate College of the Oklahoma State University.

Carrasco, M. A., y González, M. J. (2006). Aspectos conductuales de la agresión: definición y modelos explicativos. Acción Psicológica, 4(2), 7-38.

https://doi.org/10.5944/ap.4.2.478

Danish, S. J.; Nellen, V. C., \& Owens, S. S. (1996). Teaching life skills through sport: Community based programs for adolescents. En J. K. Van Raalte, \& B. W. Brewer (Eds.), Exploring sport and exercise psychology (pp. 205-225). Washington, DC: American Psychological Association.

Dias, C.; Cruz, J. F., \& Fonseca, A. M. (2014). Pre-competitive emotions in team sports: Differences across gender and perceived importance and difficulty of the competition. Revista Portuguesa de Ciências do Desporto, 14(3), 81-96.

Jones, M. (2003). Controlling emotions in sport. The Sport Psychologist, 17(4), 471-486.

Loftian, S.; Ziaee, V.; Amini, H., \& Mansournia, M. (2011). An analysis of anger in adolescent girls who practice the martial arts. International Journal of Pediatrics, 22(1), 9-14.

https://doi.org/10.1155/2011/630604

Maxwell J., \& Moores E (2007). The development of a short scale measuring aggressiveness and anger in competitive athletes. Psychology of Sport and Exercise $8(2), 179-193$.

https://doi.org/10.1016/j.psychsport.2006.03.002

Maxwell, J. P.; Visek, A. J., \& Moores, E. (2009). Anger and perceived legitimacy of aggression in male Hong Kong Chinese athletes: Effects of type of sport and level of competition. Psychology of sport and exercise, 10(2), 289-296.

https://doi.org/10.1016/j.psychsport.2008.07.010 
Miguel-Tobal, J.; Casado, M.; Cano-Vindel, A., y Spielberger, C. D. (2001). Inventario de Expresión de la Ira Estado-Rasgo STAXI-2. Madrid, España: TEA Ediciones.

Menéndez, J. I., y Fernández-Río, J. (2015). Niveles de ira en practicantes de boxeo y kickboxing: diferencias en función de la disciplina y el nivel deportivo. Cuadernos de Psicología del deporte, 15(3), 75-85.

Nicholls, A. R.; Jones, C. R.; Polman, R. C. J., \& Borkoles, E. (2009). Acute sport-related stressors, coping, and emotion among professional rugby union players during training and matches. Scandinavian Journal of Medicine \& Science in Sports, 19(1), 113-120. https://doi.org/10.1111/j.1600-0838.2008.00772.x

Nunnally, J. C., \& Bernstein, I. H. (1994). Psychometric Theory. Nueva York: McGrawHill.

Oliva, F. J., y Hernández, M. R. (2010). Validación de la versión mexicana del inventario de la expresión de ira estado-rasgo (STAXI-2). Acta Colombiana de Psicología, 13(2), 107-117.

Oliva, F. J.; Calleja, N., y Hernández, M. R. (2011). La ira en el desempeño deportivo del judoka. Revista Latinoamericana de Medicina Conductual, 1(2), 41-48. https://doi.org/10.5461/rIMC.V1.i2.24879

Proios, M. (2012). The relation between the emotion of "anger" and the cognitive processes in gymnastics athletes. International Quarterly of Sport Science, 1, 10-18.

Robazza, C., \& Bertoli, L. (2007). Perceived impact of anger and anxiety on sporting performance in rugby Players. Psychology of Sport and Exercise, 8(6), 875-896. https://doi.org/10.1016/j.psychsport.2006.07.005

Robazza, C.; Bertollo, M., \& Bortoli, L. (2006). Frequency and direction of competitive anger in contact sports. Journal of Sports Medicine and Physical Fitness, 46, 501508.

Sadeh, N.; Javdani, S.; Finy, M. S., \& Verona, E. (2011). Gender differences in emotional risk for self- and other-directed violence among externalizing adults. Journal of Consulting and Clinical Psychology, 79, 106-117.

https://doi.org/10.1037/ a0022197

Sofia, R., \& Fernando, J. (2010). As relações entre percepção de ameaça, ansiedade, raiva e agressividade no desporto: um estudo comparativo em modalidades de contacto. Revista Portuguesa de Ciências do Desporto, 13(2), 44-56.

Spielberger, C. D. (1999). Manual for the State-Trait Anger Expression Inventory, STAXI2. Odessa, FL: Psychological Assessment Resource.

Spielberger C. D.; Jacobs G.A.; Russell, S., \& Crane R.S. (1983). Assessment of anger: The state-trait anger scale. In: JN Butcher, C.D. Spielberger, (Eds.). Advances in Personality Assessment (vol. 2 pp. 159-187). Hillsdale, NJ: Lawrence Erlbaum.

Spielberger, C. D.; Johnson, E.; Russell, S.; Crane, R. S.; Jacobs, G. A., \& Worden, T. (1985). The experience and expression of anger: Construction and validation of an anger expression scale. In M. Chesney, \& R. Rosenman (Eds.). Anger and hostility in cardiovascular and behavioral disorders (pp. 5-30). Hemisphere: McGraw-Hill.

Wagstaff, C. R. D. (2014). Emotion Regulation and Sport Performance. Journal of Sport \& Exercise Psychology, 36(4), 401-412. https://doi.org/10.1123/jsep.2013-0257

Ziaee, V.; Loftian, S.; Amini, H.; Mansournia, M., \& Memari, A. (2012). Anger in adolescent boy athletes: a comparison among Judo, karate, swimming and nonathletes. Iranian Journal of Pediatrics, 22(1), 9-14. 
Menéndez-Santurio, J. I., y Fernández-Rio, J. (2017). Niveles de ira en deportistas: diferencias en función del grado de contacto y el género. RICYDE. Revista internacional de ciencias del deporte, 50(13), 356-369. https://doi.org/10.5232/ricyde2017.05003

Zimprich, D., \& Mascherek, A. (2012). Anger expression in Swiss adolescents: Establishing measurement invariance across gender in the AX scales. Journal of Adolescece, 35(4), 1013-1022.

https://doi.org/10.1016/j.adolescence.2012.02.008 